\title{
Temporal Changes Mediated by Sri Lankan Sponge Haliclona (Soestella) sp Crude Extract in in vitro Phagocytic Activity of Wistar Rats' Peritoneal Macrophages
}

\author{
Rathnayake L.M.S.M. ${ }^{1}$, Udagama P.V. ${ }^{1}$, De Silva B.G.D.N.K. ${ }^{2}$, Gunathilake K.V.K. ${ }^{2 *}$ \\ ${ }^{1}$ Department of Zoology \& Environment Sciences, University of Colombo, Sri Lanka \\ ${ }^{2}$ Department of Zoology, University of Sri Jayewardenepura, Sri Lanka \\ *kvkgunathilaka@yahoo.com
}

\begin{abstract}
Temporal changes in the production of secondary metabolites by marine organisms are fully understood. Of all marine organisms, marine sponges are ranked at the top of the hierarchy due to their ability to produce structurally novel natural secondary metabolites with bioactive properties. However, variations in the abundance of bioactive compounds produced by sponges may respond to physical constraints while the habitat spongesise, competition for space, or against fouling may also cause changes in sponge secondary chemistry. Many of these biotic or abiotic factors vary between months, seasons, and years resulting strong temporal patterns in secondary metabolites. The present study thus investigated temporal changes mediated by the crude extract of a Sri Lankan marine sponge species, Haliclona (Soestella) $s p$, on in vitro phagocytic activity of Wistar rat peritoneal macrophages (PM). Sponge samples were harvested from Unawatuna, Galle, Sri Lanka by scuba diving in 2013 and 2015, extracted with methanol/dichloromethane, followed by filtration through Whatman No 1 filter papers and rotary evaporation. The resultant crude extract of Haliclona (Soestella) sp (HSCE) was dissolved in 5\% ethanol and tested for phagocytic capacity on Wistar ratPMs at 2000, 1000, 500,100,10 $\mu \mathrm{g} / \mathrm{ml}$ concentrations by ex vivo Nitro Blue Tetrazolium assay (NBT).Absorbance was obtained as a measure of NBT dye reduction by PM cells and the phagocytic capacity of these cells was calculated. The previous results, the phagocytic activity of rat PMs evaluated ex vivo was significantly higher at HSCE doses of 10, 100 and $500 \mu \mathrm{g} / \mathrm{ml} \quad(>1 ; \mathrm{P}<0.01)$ than at $1,000 \& 2,000 \mu \mathrm{g} / \mathrm{ml}$ doses $(<1 ; \mathrm{P}<0.05)$, indicative of higher stimulation of PM cells by lower doses of the HSCE extracted from sponges collected in 2013. However, a dose dependent increase in phagocytic activity by PM cells was observed by HSCE extracted from sponge samples collected in $2015 \quad(\mathrm{P}<0.05)$, indicative of the higher stimulation of PM cells by higher doses of the HSCE extracted from sponges collected in 2015. Therefore, it is evident that a temporal change had occurred in the secondary metabolites responsible for phagocytic activity produced by Haliclona (Soestella) sp within a periods of two years (from 2013 -2015).
\end{abstract}

Keywords: Temporal change, Haliclona (Soestella sp), Phagocytic activity

Proceedings of the $22^{\text {nd }}$ International Forestry and Environment Symposium 2017 of the Department of Forestry and Environmental Science, University of Sri Jayewardenepura, Sri Lanka 